\title{
Antioxidative mechanism and anisotropic charge transport properties of mangiferin: A theoretical study
}

\author{
Chunyuan $\mathrm{Hou}^{a, b *}$, Guoping Chai ${ }^{a}$ and Haijun $\mathrm{Li}^{a}$ \\ a Department of Biochemical Engineering, Anhui Polytechnic University, Wuhu \\ 241000, China \\ ${ }^{b}$ State Key Laboratory of Molecular Reaction Dynamics, Dalian Institute of Chemi- \\ cal Physics, Chinese Academy of Sciences, Dalian 116023, China
}

Received 22 December 2014; Accepted (in revised version) 25 Februrary 2015

Published Online 1 March 2015

\begin{abstract}
A quantum mechanical approach has been used to investigate the antioxidative mechanism for scavenging $\bullet \mathrm{OOH}$ and $\bullet \mathrm{OH}$ radicals using mangiferin in solution phase. Mangiferin is also used as a potent applicant for organic semiconductor. The charge-transport properties in mangiferin have been studied based on the first-principle DFT combined with the Marcus-Hush theory. Density functional theory (DFT) calculations at the B3LYP / 6-311+G(d,p) level were used to optimize mangiferin and its different forms. The lowest theoretical bond dissociation enthalpy (BDE) value for 7-OH site of mangiferin in water, indicates that 7-OH group is important in the antioxidant activity. The relative values of enthalpies also show that oxidation of mangiferin by $\bullet \mathrm{OOH}$ and $\bullet \mathrm{OH}$ radical is an exothermic process. The predicted maximum electron mobility value of mangiferin is $0.148 \mathrm{~cm}^{2} \mathrm{~V}^{-1} \mathrm{~s}^{-1}$, which appears at the orientation angle near $49^{\circ} / 311^{\circ}$ of conducting channel on the reference planes ab. Theoretical investigation of natural semiconductors is helpful for designing higher performance electronic materials used in biochemical and industrial field to replace expensive and rare organic materials.
\end{abstract}

PACS: $34.70 .+\mathrm{e} ; 42.70 . \mathrm{Nq}$

Key words: anisotropic, organic semiconductor, mangiferin, antioxidant, charge transport.

\section{Introduction}

Mangiferin is a class of polyphenolic compounds, having two benzene ring (A and B) condensed with a six-membered ring $(C)$ (see Fig. 1). Its presence has been investigated in mango, hypericum elegans, and tripterospermum lanceolatum. Mangiferin have attracted attention of many researchers since 1960' because of its widerange appliactions in

${ }^{*}$ Corresponding author. Email address: cyhou@ahpu.edu.cn (C.-Y. Hou) 


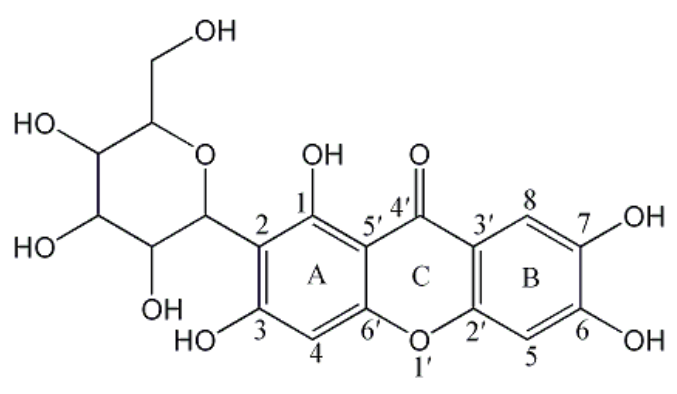

Figure 1: Chemical structure of mangiferin.

biochemical industries [1-9]. Mangiferin bioactivities have been correlated with radical scavenging as well as inhibition of oxidative stress and complex formation with Fe [8,9]. Some natural polyphenolic compounds, such as 3-hydroxyflavone and quercetin, have been used as an additive in solar cell devices [10-12] due to its low cost, easy attainability, and no environmental threat. Mangiferin have become a viable alternative to expensive and rare organic materials. Therefore, in order to elucidate its pharmacological mechanism; use as novel drugs and natural electronic materials investigation of its fundamental chemical behavior and electronic characteristics will be of great significance. Polyphenolic compounds, such as flavonoids, have also been found as potential anti-cancer, antiinflammatory, antibacterial, antiviral, and antiallergic applicants [13-17]. They prevent biological and chemical substances from oxidative damage by reactive oxygen species and have been used as possible therapeutics against a wide variety of diseases caused by radical damage [15]. Recently, the antimutagenic activity of phenolic compounds, particularly flavonoids such as rutin, have been studied on the basis of their scavenging capability against the free radicals, such as $\bullet \mathrm{OH}$ [16]. The reaction can be expressed as $\mathrm{R}^{\bullet}+\mathrm{R}^{\prime} \mathrm{OH} \rightarrow \mathrm{R}^{\prime} \mathrm{O}^{\bullet}+\mathrm{RH}$, the formed radical $\mathrm{R}^{\prime} \mathrm{O}^{\bullet}$ should be relatively stable so that the reaction becomes thermodynamically favorable $[18,19]$.

Many experimental and theoretical studies about antioxidant properties of polyphenolic compounds have focused on the investigation of flavonoids [18-24]. Recently Lengyel et al. [18] and Vaganek et al. [19] have carried out calculations for O-H bond dissociation enthalpies, proton dissociation enthalpies, ionization potentials, proton affinities and electron transfer enthalpies related to HAT, SET-PT and SPLET mechanisms in gas and solution-phases of some isoflavones and flavonoids. The electrical anisotropy as an intrinsic property of organic semiconductors has aroused the attention of many researchers [25-30] A strong anisotropy of the field-effect mobility with the a-b plane of single crystals of rubrene has been observed by Sundar et al. [28]. Direct and inverse photoemission spectroscopy and some other experimental methods have been performed to study the n-type doping of a variety of electron transport materials by Kahn and coworkers [31-36]. Although there are several computational studies for the electrical anisotropy [37-40], a systemic investigation of anisotropic mobilites is still lacking. Based on Marcus-Hush theory [41,42], Han and coworkers have developed a method to simulate the angular res- 
olution anisotropic mobility, which correlates with the underlying electronic properties and the molecular packing [43-49].

Many researchers have carried out fabrications and electrical/optical characterizations of photovoltaics using natural products thus replacing organic semiconductors due to its low cost, abundant supply of raw materials, feasibility and eco-friendly nature [1012]. Among above mentioned, quercetin and 3-hydroxyflavone were considered potent applicants for organic semiconductor devices fabrication $[10,11]$. However, there is little report on mangiferin another important class of polyphenolic compounds, as electronic materials upto now. No literature has been found to understand the anisotropic mobility that correlates with the underlying electronic properties and the molecular packing. Because the experimental results demonstrate that some polyphenolic compounds can be used as promising class of semiconductors for organic electronics and optoelectronics, we have theoretically investigated the charge mobilities of mangiferin and simulate the angular-resolution mobilities. In order to design high-performance semiconducting materials, we have been motivated to make a full and accurate theoretical investigation on mangiferin about the relationship between the charge-transport properties and the molecular packing. Only two molecular models of mangiferin have been considered in this article. The crystal structures can be found in ref [2], the crystal structures of mangiferin demonstrated distinct stacking modes.

The aim of this work is to investigate the anisotropic charge transport in mangiferin and antioxidant mechanism of mangiferin, including scavenging activities against the $\bullet \mathrm{OOH}$ and $\bullet \mathrm{OH}$ free radicals. In the present work, DFT method at B3LYP/6-311+G(d,p) level has been used to optimize the geometric structures of all reactants, transition states, intermediates, and products. The reaction mechanisms of $\bullet \mathrm{OH}$ and $\bullet \mathrm{OOH}$ radicals attacking mangiferin in solution are clarified. In addition, the effective electronic couplings and charge transport mobilities have been deduced based on first principles quantum mechanics calculations combined with Marcus-Hush theory [41,42], the anisotropic charge transport of mangiferin is also investigated.

\section{Computational details}

\subsection{Theoretical parameters: calculation of the bond-dissociation energy and spin distribution}

Bond dissociation enthalpy (BDE) is a quantum chemically determined suitable parameter for describing the abstraction of a hydrogen radical from an $\mathrm{O}-\mathrm{H}$ bond. It can be calculated by the difference in total enthalpies between mangiferin and its corresponding radical, $\mathrm{E}_{B}=\mathrm{H}_{h}+\mathrm{H}_{r}-\mathrm{H}_{p}$, where $\mathrm{H}_{h}$ is the enthalpy of the $\mathrm{H}$-atom, $\mathrm{H}_{r}$ is the enthalpy of the radical generated by $\mathrm{H}$-abstraction, and $\mathrm{H}_{p}$ is the enthalpy of the parent molecule. Previous theoretical results of the BDE in phenolic compounds show the DFT method with 6-311+G $(d, p)$ basis set [50] is sufficient to give an accurate description of the BDE [51-56]. Hence, this method is used for the continuation of our studies. Because the DFT 
method does not provide enthalpies directly, the total enthalpies of the species $R^{\bullet}, H(R)$, at the temperature $\mathrm{T}$ are usually expressed as $\mathrm{H}(\mathrm{R})=\mathrm{E}_{0}+\mathrm{ZPE}+\Delta \mathrm{H}_{\text {trans }}+\Delta \mathrm{H}_{\text {rot }}+\Delta \mathrm{H}_{\text {vib }}$ $+\mathrm{RT}$, where $\mathrm{E}_{0}$ is the total electronic energy, $\mathrm{ZPE}$ represents zero-point energy, $\Delta \mathrm{H}_{\text {trans }}$, $\Delta \mathrm{H}_{\text {rot }}$ and $\Delta \mathrm{H}_{v i b}$ stand for the translational, rotational and vibrational contributions to the enthalpy. Finally, RT is PV-work term and is added to convert the energy to the enthalpy $[17,18]$. The computed value of $\mathrm{H}$-atom enthalpy is $-314.5 \mathrm{kcal} / \mathrm{mol}$ at $\mathrm{T}=298 \mathrm{~K}$.

Spin distributions is another important parameter of delocalization possibilities, which is relevant to the antioxidant activity. We have also calculated the spin distributions for the different radicals of mangiferin. The $\bullet \mathrm{OH}$ radical in the cells can easily cross cell membranes at specific sites and reacts with most biomolecules, which is the reason of tissue damage and cell death. Then, the mechanism for the radical-scavenging reactions for mangiferin is discussed.

\subsection{Molecular orbital calculation}

All the calculations for the optimizations of all the stationary points are performed with the Gaussian 03 program [57] at B3LYP / 6-311+G(d,p) level of theory on a Lenovo server. Harmonic vibrational frequencies are analyzed at the same level of optimization. There are no imaginary frequencies for the stationary points as true minima and only one imaginary frequency for the transition states. In order to simulate the reaction in solution, PCM model is implemented to optimize structures, in which the dielectric constant 78.39 at $298.15 \mathrm{~K}$ for $\mathrm{H}_{2} \mathrm{O}$ is used [58-60]. $\Delta \mathrm{E}_{0}$ is the zero-point energy (ZPE) corrected relative electronic energy. Intrinsic reaction coordinate (IRC) calculations at the same level are performed to correct the transition states between designated local minima.

\subsection{Reorganization energy}

The reorganization energy usually consists of the internal and external contributions. The molecular geometric relaxation leads to the internal reorganization energy, and the external reorganization energy is induced by polarization of the surrounding molecules. The external reorganization energy in organic crystals is usually neglected because of the low dielectric constants of molecular solids, thus only the internal reorganization energy is considered $[61,62]$. The reorganization energy $\lambda$ can be calculated by using the adiabatic potential energy surface method [63-65], which can be expressed as Eqs. 1-3.

$$
\begin{aligned}
& \lambda=\lambda_{i}^{(1)}+\lambda_{i}^{(2)} \\
& \lambda_{i}^{(1)}=E_{H}-E_{B} \\
& \lambda_{i}^{(2)}=E_{F}-E_{C}
\end{aligned}
$$

Here $E_{B}$ and $E_{C}$ represent the energies of the neutral and cation/anion molecules in their lowest energy geometries, respectively; $\mathrm{E}_{F}$ and $\mathrm{E}_{H}$ are the energies of the neutral and cation/anion monomers with the geometries of the cation/anion and neutral species, 
respectively; $\lambda_{i}^{(1)}$ means the geometry relaxation energy of one neutral molecule from the most stable geometry of the ionic state to the lowest energy geometry of the neutral state, and $\lambda_{i}^{(2)}$ means the geometry relaxation energy of one cation/anion molecule from the most stable geometry of the neutral state to the lowest energy geometry of the ionic state [63]. Electron affinity and ionization potential can be related to the ability of the electron injecting into empty LUMOs from the metal electrode and the electron injecting into the metal electrodes from HOMOs, respectively. Afterward, the adiabatic ionization potential (IP) and electron affinities (EA) are calculated by the following equations

$$
\begin{aligned}
& I P=E_{C}(+)-E_{B} \\
& E A=E_{C}(-)-E_{B}
\end{aligned}
$$

\subsection{Intermolecular electronic coupling}

The intermolecular electronic coupling $\mathrm{V}$ is another important parameter of charge transfer capability of materials, which is related to the molecular orbitals of the conjugated molecules. The method of calculating the intermolecular electronic coupling $\mathrm{V}$ of each dimer in organic semiconductors can be found in refs [66-67]. We select the geometries for dimer calculations from the observed X-ray crystal structure. The intermolecular electronic coupling $\mathrm{V}$ is calculated directly by the spatial overlap $\left(\mathrm{S}_{i j}\right)$, charge-transfer integral $\left(\mathrm{V}_{i j}\right)$, and site energies $\left(\mathrm{H}_{i i}, \mathrm{H}_{j j}\right)$, which can be written as

$$
\begin{aligned}
& V=\frac{V_{i j}-S_{i j}\left(H_{i i}+H_{j j}\right) / 2}{1-S_{i j}^{2}} \\
& V_{i j}=\left\langle\phi_{i}^{H / L}|\hat{H}| \phi_{j}^{H / L}\right\rangle \\
& \left\langle\phi_{i} \mid \phi_{j}\right\rangle=s_{i j} \\
& H_{i j}=\left\langle\phi_{i}^{H / L}|\hat{H}| \phi_{i}^{H / L}\right\rangle
\end{aligned}
$$

where $H$ represents the dimer system Kohn-Sham Hamiltonian, and $\phi_{i(j)}$ is the monomer HOMOs (for hole transport) or LUMOs (for electron transport) with Lowdin's symmetric transformation, which can be used as the orthogonal basis set for calculation [67]. The $\mathrm{V}$ calculations in mangiferin dimmers is implemented by Gaussian 03 at the PW91PW91/ 6$311+\mathrm{G}(\mathrm{d}, \mathrm{p})$ level $[67,68]$.

\subsection{Hopping rate and angular resolution anisotropic mobility}

In organic semiconductors, based on the Marcus-Hush theory $[41,42,69,70]$, the transport of hole/electron is calculated by means of charge carrier hopping between neighboring molecules at room temperature. The hopping rate $(k)$ [71-73] can be expressed as

$$
k=\frac{2 \pi}{h}|V|^{2} \sqrt{\frac{\pi}{k_{B} T \lambda}} \exp \left(-\frac{\lambda}{4 k_{B} T}\right)
$$


where $\mathrm{V}$ stands for the effective electronic coupling between neighboring molecules, $\lambda$ represents the reorganization energy, $k_{B}$ is the Boltzmann constant, and $T$ is the temperature, which is $300 \mathrm{~K}$ in the calculations. It is assumed that there is no correlation between charge hopping motions and the hopping motion is a homogeneous random walk, $[43,45,72]$ afterward the diffusion coefficient (D) caused by the hopping rate is expressed as eq

$$
D=\lim _{t \rightarrow \infty} \frac{1}{2 n} \frac{\left\langle x(t)^{2}\right\rangle}{t} \approx \frac{1}{2 n} \sum_{i} r_{i}^{2} k_{i} P_{i}=\frac{1}{2 n} \frac{\sum_{i} r_{i}^{2} k_{i}^{2}}{\sum_{i} k_{i}}
$$

where $\mathrm{n}$ means the spatial dimensionality, $\mathrm{i}$ is the ith pathway, $r_{i}$ represents the intermolecular center-of-mass distance between two neighboring molecules, $k$ is the intermolecular hopping rate, and $P_{i}$ stands for the hopping probability calculated by eq

$$
P_{i}=\frac{k_{i}}{\sum_{i} k_{i}}
$$

Based on the Einstein relation, in organic semiconductors the drift mobility for charge carrier (hole/electron) transport is calculated by eq

$$
\mu=\frac{e D}{k_{B} T}
$$

It shows that the rate of charge hopping depends on two microscopic parameters: the effective electronic coupling $V$ and the reorganization energy $\lambda$. So, in order to improve the charge mobility in organic semiconductor materials, many attempts of optimizing these two parameters have been made [74-76]. The specific surface of organic crystal leads to the magnitude of the field-effect mobility in a particular transistor channel. Therefore, the anisotropic mobility of charge transport in organic semiconductors is an intrinsic property [28]. Han et al. presented a model to simulate the anisotropic mobility $(\mu \phi)$ by projecting the different hopping pathways $[43,45]$. The equation of angular resolution anisotropic mobility can be expressed as

$$
\mu_{\phi}=\frac{e}{2 k_{B} T} \sum_{i} k_{i} r_{i}^{2} P_{i} \cos ^{2} \gamma_{i} \cos ^{2}\left(\theta_{i}-\phi\right)
$$

The intermolecular packing in the organic single crystals is descripted by parameters $r_{i}, \gamma_{i}$, and $\theta_{i}$, which is the ith hopping distance, the angle of the ith hopping pathway relative to the transport plane of the organic crystal molecular stacking layer, and the orientation angle of the projected electronic coupling pathways of different dimer types, respectively. $\phi$ is defined as the conducting channel relative to the same reference axis (generally using the crystallographic axis). Thus, the angle between the different pathways and the conducting channel is $\theta_{i}-\phi . \quad P_{i}$ and $k_{i}$ are calculated by eqs 10 and 12, respectively. For the hopping pathways on the basal transport stacking layer in the organic crystal, the values of $\gamma_{i}$ are $0^{\circ}$. Eq. 14 gives an analytic function to calculate the 
Table 1: Some structural details of the optimized structure of mangiferin at the B3LYP/6-311+G(d,p) level.

\begin{tabular}{|c|c|c|c|c|c|}
\hline \multicolumn{2}{|c|}{ Bond distance $(\AA)$} & \multicolumn{2}{|c|}{ Bond angles $\left(^{\circ}\right)$} & \multicolumn{2}{c|}{ Dihedral angles $\left({ }^{\circ}\right)$} \\
\hline O-1-H-1 & 0.99446 & C-1-O-1-H-1 & 107.27 & C-5-C-1-O-1-H-1 & 0.34 \\
\hline O-3-H-3 & 0.96288 & C-3-O-3-H-3 & 109.91 & C-4-C-3-O-3-H-3 & -0.83 \\
\hline O-6-H-6 & 0.96727 & C-6-O-6-H-6 & 108.60 & C-5-C-6-O-6-H-6 & -0.04 \\
\hline O-7-H-7 & 0.96259 & C-7-O-7-H-7 & 110.77 & C-8-C-7-O-7-H-7 & -0.02 \\
\hline
\end{tabular}

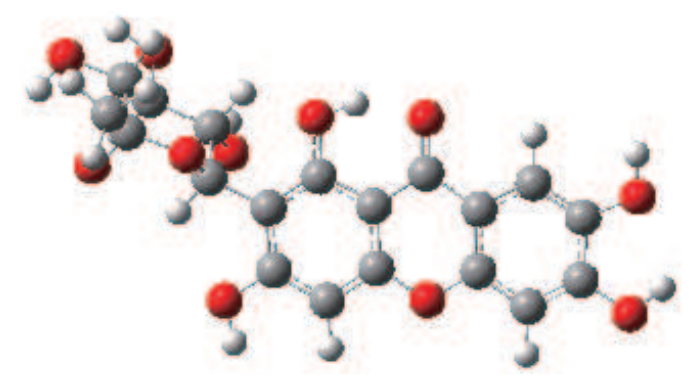

Figure 2: Optimized structure of mangiferin in $\mathrm{H} 2 \mathrm{O}$ solvent at the $\mathrm{B} 3 \mathrm{LYP} / 6-311+\mathrm{G}(\mathrm{d}, \mathrm{p})$ level.

angular resolution anisotropic mobilities of any type of organic semiconductors by connecting the crystal packing and electron coupling $V$ with the outer measuring channel angle $\phi$. One molecule $\mathrm{M}$ as the initial position is arbitrarily chose for the charge transport. The intermolecular electronic couplings with all neighboring molecules in a dimer model are calculated. In organic crystals, neighboring molecules can be characterized as transverse dimer T, parallel dimer P, and longitudinal dimer L. In this work, the charge transport is under the ideal conditions at room temperature. The electronic coupling between the molecules in the same organic packing layer is much stronger than that between the molecules in two adjacent molecular stacking layers. The electronic coupling of the head-to-tail stacking ( $\mathrm{L}$ dimmers) is very small, and thus the charge transport between layers (L dimer) is less efficient and negligible, which means that the charge transport in crystals is $2 \mathrm{D}$ transport.

\section{Results and discussion}

\subsection{Geometry optimization of mangiferin and energy properties}

Results of rotameric distributions in the previous study [2] show that the gauche-gauche conformer is the most abundant form of mangiferin in gas phase and water. The molecular geometries of mangiferin in neutral and charged state are fully optimized at DFT with B3LYP $/ 6-311+g(d, p)$ level in gas phase to calculate the reorganization energies based on the adiabatic potential energy surface. The geometry of neutral molecular is also optimized by using PCM to consider the solvent effect, which is shown in Fig. 2.

A selection of calculated bond distances, bond angles, and dihedral angles is listed in 
Table 2: Calculated ionization potential (IP), electron affinity (EA), and molecular frontier orbital energies (HOMO and LUMO) as well as energy gap (H-L gap) with the method B3LYP/6-311+g(d,p).

\begin{tabular}{|c|c|c|c|c|}
\hline $\mathrm{IP}(\mathrm{eV})$ & $\mathrm{EA}(\mathrm{eV})$ & $\mathrm{HOMO}(\mathrm{eV})$ & $\mathrm{LUMO}(\mathrm{eV})$ & H-L gap $(\mathrm{eV})$ \\
\hline 7.034 & 0.629 & -6.177 & -2.068 & 4.109 \\
\hline
\end{tabular}

Table 3: The calculated relaxation energies $\lambda i^{(1)}$ and $\lambda i^{(2)}$ and as well as reorganization energies $\lambda$ in mangiferin (in electronvolts).

\begin{tabular}{|c|c|c|c|c|c|}
\hline \multicolumn{3}{|c|}{ Electron transfer } & \multicolumn{3}{c|}{ Hole transfer } \\
\hline$\lambda i^{(1)}$ & $\lambda i^{(2)}$ & $\lambda$ & $\lambda i^{(1)}$ & $\lambda i^{(2)}$ & $\lambda$ \\
\hline 0.400 & 0.588 & 0.988 & 0.150 & 0.182 & 0.332 \\
\hline
\end{tabular}
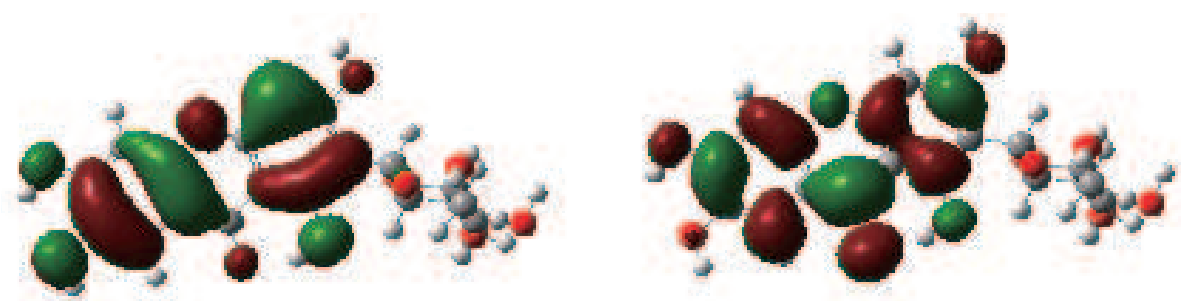

Figure 3: Presentation of HOMO (left) and LUMO (right) orbital of the mangiferin molecule.

Table 1. It can be seen that there is apparent conjugation effect, and the distance of $\mathrm{O}-1-\mathrm{H}-$ 1 is about $0.032 \AA$ greater than $\mathrm{O}-3-\mathrm{H}-3$, which indicates the internal hydrogen bonding on the $\mathrm{H}-1$ atom. The ortho-dihydroxy structure in the $\mathrm{B}$ ring confers high stability to the radical species by $\mathrm{H}$-bond formation.

The HOMO and LUMO distribution of mangiferin molecule is presented in Fig. 3. The calculated energy gap between the HOMO and LUMO of molecule ( $\triangle E_{\text {gap }}=E_{L U M O}-$ $\left.E_{H O M O}\right)$ of the mangiferin molecule is about $4.10 \mathrm{eV}$, which indicates that the molecule is suitable to be an used as antioxidant and promising candidate as organic semiconductor $[48,77,78]$. The calculated IP, EA as well as the molecular frontier orbital energies (HOMO and LUMO) are summarized in Table 2. The suitable IP value of mangiferin ensures high stability of organic material, and the efficient injection of electrons into the metal electrode from $\mathrm{HOMO}$, thus mangiferin is suitable to be used as p-type semiconductor. In comparison, the low EA value hinders its electron accepting properties.

The calculated results of relaxation energies $\lambda i^{(1)}$ and $\lambda i^{(2)}$ as well as reorganization energies $\lambda$ for mangiferin are presented in Table 3. Because high reorganization energy is not helpful for higher carriers transport [76], mangiferin is suggested to prefer to function as a high efficiency p-type semiconductor rather than the n-type semiconducting materials only in the view of $\lambda$ values. 
Table 4: Calculated values of the BDE for different $\mathrm{OH}$ positions in mangiferin at the UB3LYP/6-311+G(d,p) level.

\begin{tabular}{|l|c|c|c|c|}
\hline $\mathrm{OH}$ position & $1-\mathrm{OH}$ & $3-\mathrm{OH}$ & $6-\mathrm{OH}$ & $7-\mathrm{OH}$ \\
\hline $\mathrm{BDE}(\mathrm{kcal} / \mathrm{mol})$ & 97.5 & 96.2 & 90.2 & 85.9 \\
\hline
\end{tabular}

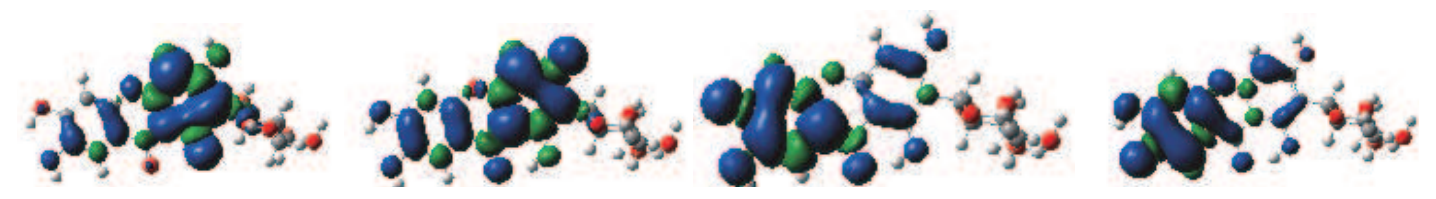

Figure 4: Spin density plot calculated by the Mulliken spin population analysis for the molecule after $\mathrm{H}$-atom was removed from the $1,3,6$, and $7-\mathrm{OH}$ positions(from left to right).

\subsection{BDE values of optimized mangiferin}

The radicals formed by hydrogen abstraction from 1, 3, 6, and 7-OH positions of mangiferin are optimized using the UB3LYP/6-311+G(d,p) method in aqueous solvation sphere. The calculated BDE values for the four radicals are listed in Table 4.

The results indicate that BDE increases in following order for different positions of $\mathrm{OH}$ groups: $7-\mathrm{OH}<6-\mathrm{OH}<3-\mathrm{OH}<1-\mathrm{OH}$. BDE value for the $1-\mathrm{OH}$ group was highest and found to be $97.5 \mathrm{kcal} / \mathrm{mol}$, which indicates that internal hydrogen bonding in this case is thermodynamically unfavorable. These results clearly show that $\mathrm{H}$ transfer is more energetically favorable from the B-ring, especially from the 7-OH position. Therefore, the 7-OH is the most important site for $\mathrm{H}$-transfer and consequently for the antioxidant capacity. Mangiferin with the dihydroxy functionality is also expected to act as hydrogen donors because of the calculated low BDE values. The BDE value for the 7-OH of mangiferin is close to apigenin, fisetin, luteolin, quercetin and norathyriol [79] but a little bit greater than cyanidin and epicatechin [19].

\subsection{Comparison between the spin densities of the radicals formed from the B- and A-rings}

The stabilization of the radicals obtained after $\mathrm{H}$-abstraction are caused by $\pi$-electron delocalization. Fig. 4 shows the calculated electron distributions plots in the highest occupied molecular orbital (HOMO) of the four radicals, which are obtained to understand the relationship between electron delocalization and the reactivity of the radicals. The HOMO shape is similar for the four radicals, and no sufficient variations for explaining the differences in activity between those $\mathrm{OH}$ groups can be seen directly. The spin density is often considered to be a more realistic parameter, which can provide a better representation of the reactivity [80]. Leopoldini et al. have pointed out the importance of the spin density for the description of polyphenolic compounds [21]. We have calculated net charges and spin distribution of the four radicals, which are presented in Table 5. It indicates that the spin population on the remaining $\mathrm{O}$ atom after $\mathrm{H}$-removal and on 
Table 5: omparison between derived net atomic charges and unpaired spin distributions for different radicals of mangiferin at the UB3LYP/6-311+G(d,p) level .

\begin{tabular}{|l|c|c|c|c|}
\hline Radical position Atom & $\begin{array}{c}\text { O-1 } \\
\text { Spin(charge })^{a}\end{array}$ & $\begin{array}{c}\text { O-3 } \\
\text { Spin (charge })^{a}\end{array}$ & $\begin{array}{c}\text { O-6 } \\
\text { Spin(charge })^{a}\end{array}$ & $\begin{array}{c}\text { O-7 } \\
\text { Spin(charge })^{a}\end{array}$ \\
\hline C-1 & $-0.07(0.17)$ & $-0.08(0.37)$ & $0.02(0.35)$ & $0.01(0.41)$ \\
\hline C-2 & $0.43(0.16)$ & $0.19(0.33)$ & $-0.01(0.12)$ & $0.01(0.08)$ \\
\hline C-3 & $-0.17(-0.13)$ & $-0.08(-0.45)$ & $0.02(-0.26)$ & $0(-0.13)$ \\
\hline C-4 & $0.43(-0.45)$ & $0.38(-0.37)$ & $0(-0.53)$ & $0.03(-0.49)$ \\
\hline C-5 & $0(-0.43)$ & $-0.02(-0.45)$ & $0.14(-0.55)$ & $-0.12(-0.34)$ \\
\hline C-6 & $0(-0.22)$ & $0.03(-0.18)$ & $-0.01(0.05)$ & $0.19(-0.24)$ \\
\hline C-7 & $0.03(-0.17)$ & $0.03(-0.18)$ & $0.30(-0.22)$ & $0.03(-0.02)$ \\
\hline C-8 & $-0.02(-0.06)$ & $-0.02(-0.01)$ & $-0.12(0.01)$ & $0.23(-0.03)$ \\
\hline C-2' & $0.01(-0.86)$ & $0.02(-0.82)$ & $-0.08(-0.64)$ & $0.21(-0.81)$ \\
\hline C-3' & $0.03(1.56)$ & $0.04(1.62)$ & $0.33(1.65)$ & $-0.01(1.66)$ \\
\hline C-4' & $0(-0.11)$ & $-0.05(0.01)$ & $-0.04(0)$ & $0.01(-0.10)$ \\
\hline C-5' & $0.12(0.95)$ & $0.32(1.05)$ & $0.02(0.99)$ & $0(0.88)$ \\
\hline C-6' & $-0.08(-0.55)$ & $-0.14(-0.97)$ & $-0.01(-1.08)$ & $-0.01(-0.91)$ \\
\hline O-1 & $0.29(-0.27)$ & $-0.01(-0.37)$ & $0.01(-0.35)$ & $0.01(-0.35)$ \\
\hline O-3 & $-0.01(-0.27)$ & $0.34(-0.31)$ & $0.01(-0.25)$ & $0(-0.25)$ \\
\hline O-6 $^{\prime}$ & $0(-0.30)$ & $0.01(-0.28)$ & $0.28(-0.38)$ & $0.05(-0.24)$ \\
\hline O-7 & $0.01(-0.37)$ & $0.01(-0.36)$ & $0.10(-0.25)$ & $0.33(-0.41)$ \\
\hline O-1' & $0.03(-0.03)$ & $0.01(-0.04)$ & $0(-0.06)$ & $0.04(-0.02)$ \\
\hline O-4' & $0(-0.39)$ & $0.04(-0.39)$ & $0.05(-0.41)$ & $0.01(-0.40)$ \\
\hline
\end{tabular}

${ }^{a}$ Spin: unpaired spin density on the atomic center; charge: net atomic charges.

the neighboring $\mathrm{C}$-atoms appears to be slightly less delocalized issued from the A-ring than those located on the B-ring (6-OH and 7-OH positions). For example, for the 1-OH mangiferin radical the spin density populates mainly on the O-atom in the O-1. Generally speaking, the less delocalized the spin density in the radical leads more difficultly to the formation of the radical and makes the BDE higher [81]. As a consequence, the BDE is higher in the A-ring than in the B-ring.

\section{4 $\bullet \mathrm{OH}$ and $\bullet \mathrm{OOH}$ radical scavenging mechanism}

Since the scavenging mechanisms of mangiferin reacting with free radicals are not fully understood, afterward the reaction paths when the $\bullet \mathrm{OH}$ and $\bullet \mathrm{OOH}$ radical attack the mangiferin molecule are studied.

It is very important to remove $\bullet \mathrm{OH}$ for the protection of living systems. Since mangiferin is expected to act as hydrogen donor, reactants and products are mangiferin with $\bullet \mathrm{OH}$ radical and the corresponding phenoxy radical with $\mathrm{H}_{2} \mathrm{O}$, respectively. We optimize the geometries of the products and reactants at the UB3LYP / 6-311+G(d,p) level in water. No 


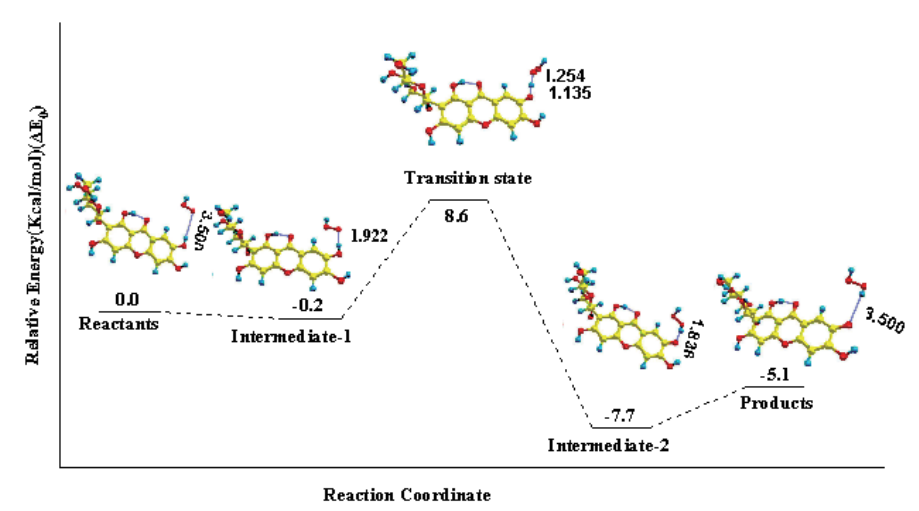

Figure 5: Relative energy profile for the reaction of $\bullet \mathrm{OOH}$ radical attack to the mangiferin molecule.

transition state geometry between products and reactants can be found. Therefore, it is expected that the $\bullet \mathrm{OH}$ directly attacks on the oxygen atom adjacent to $\mathrm{C}-1,3,6,7$. From the discussion on BDE and charge density distribution, the 7-OH is considered to be the most suitable site that $\bullet \mathrm{OH}$ can attack, the relative energy value is obtained, which suggests that this reaction is exothermic by about $39.8 \mathrm{kcal} / \mathrm{mol}$. It is thermodynamically in advantage of antioxidant capacity compared with the hydroxyl radical scavenging of rutin [20].

Compared with $\bullet \mathrm{OH}$ radical, as an important route in the oxidative injury to tissue, the peroxyl radical $(\bullet \mathrm{OOH})$ is more difficult to react with phenolic compounds. To gain an insight into the antioxidant activity, then the mechanism for the $\bullet \mathrm{OOH}$ radical reacting with mangiferin is discussed. Furthermore, as a "site-specific" interaction, hydrogen boding is important to understand microscopic structures and functions in many molecular and supramolecular systems, such as organic compounds in solvent, proteins, and DNA $[82,83]$. Afterward, the $\bullet \mathrm{OOH}$ radical approaching the mangiferin molecule from the 7-OH site is studied. We optimize the geometries of the reactants, transition states, intermediates and the products at the UB3LYP / 6-311+G(d,p) level in water. Intermediate-1 and intermediate- 2 represent the intermolecular and intramolecular hydrogen bonds in mangiferin with $\bullet \mathrm{OOH}$ radical and the corresponding phenoxy radical with $\mathrm{H}_{2} \mathrm{O}_{2}$, respectively. The reaction starts from the formation of intermediate- 1 and then the $7-\mathrm{OH}$ homolytic bond cleavage in intermediate- 1 to produce intermediate- 2 , finally leading to the products of $\mathrm{H}_{2} \mathrm{O}_{2}$ and the phenoxy radical (Fig. 5). The transition state between intermediate- 1 and intermediate- 2 is optimized. The relative energy values suggest that the reaction is also exothermic and about $5.1 \mathrm{kcal} / \mathrm{mol}$ energy is released. In addition, the products are generated from intermediate- 2 by passing over a transition state with a barrier of $8.6 \mathrm{kcal} / \mathrm{mol}$. The small barrier suggests the instant conversion of intermediate- 1 to intermediate- 2 and then to the products.

It is expected that mangiferin is oxidized by radicals, which leads to a more stable, less reactive radical. The study of mangiferin is complicated due to the scarcity of data on bioavailability, so insufficient methods are available to measure oxidative damage in 
Table 6: Calculated overlap $\mathrm{S}$ and electronic couplings $\mathrm{V}$ for hole transport $(\mathrm{eV})$ and corresponding entermolecular center-of mass distanes $r(\AA)$ for different hopping pathways ${ }^{a}$.

\begin{tabular}{|l|c|c|}
\hline pathway & $\mathrm{r} \AA$ & $\mathrm{V}(\mathrm{eV})$ \\
\hline $\mathrm{T}$ & 4.82 & 0.053 \\
\hline $\mathrm{T}_{1}$ & 11.21 & 0.001 \\
\hline $\mathrm{T}_{2}$ & 14.83 & 0 \\
\hline $\mathrm{P}$ & 8.83 & 0.001 \\
\hline $\mathrm{T}_{2}{ }^{\prime}$ & 8.83 & 0 \\
\hline $\mathrm{P}^{\prime}$ & 14.83 & 0.001 \\
\hline Maximum mobility & \multicolumn{2}{|c|}{$0.148 \mathrm{~cm}^{2} \mathrm{~V}^{-1} \mathrm{~s}^{-1}$} \\
\hline
\end{tabular}

${ }^{a}$ Simulated maximum mobilities are given in the last line $(\mathrm{T}=300 \mathrm{~K})$.
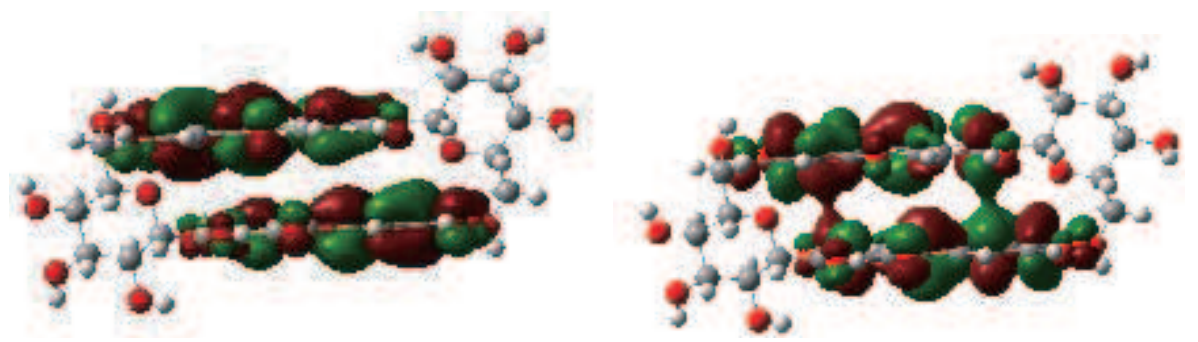

Figure 6: Molecular packings and charge hopping pathways in mangiferin with the center-of-mass distance and the angle of the projected electronic coupling pathways relative to the reference axis.

vivo.

\subsection{Effective electronic coupling and anisotropic charge transport mobility}

For mangiferin crystal structure, we can define six types of intermolecular packing modes as $\mathrm{P}, \mathrm{P}^{\prime}, \mathrm{T}, \mathrm{T}_{1}, \mathrm{~T}_{2}$ and $\mathrm{T}_{2}^{\prime}$ and the angles between the hopping pathways and reference axis can be seen in Fig. 6, which are labeled as $\theta_{P}, \theta_{P}{ }^{\prime}, \theta_{T}, \theta_{T 1}, \theta_{T 2}$, and $\theta_{T 2}{ }^{\prime}$, respectively. The orientation angle of the conducting channel relative to the reference axis is $\phi$. Fig. 7 shows that mangiferin have antiparallel geometries. The molecular packing diagram in Fig. 6 shows that mangiferin exhibits a face-to-face $\pi$-stacking along the a axis. The effective intermolecular electronic couplings $\mathrm{V}$ for hole (HOMO) transport in these six cases are listed in Table 6. Moreover, intermolecular center-of-mass distances of various packing modes can be seen. Because the stacking modes with larger orbital overlap and shorter distance are favored for the large electronic coupling, we can see that $\mathrm{T}$ dimer is the most important electron-transport pathways. The T direction is the dominant conducting channel, which has the largest electronic coupling of $0.053 \mathrm{eV}$ and shortest intermolecular distance of $4.82 \AA$. It indicates that the parallel packing mode usually yields larger coupling term than other packing because the cofacial stacking structure is expected to offer more efficient orbital overlap. 

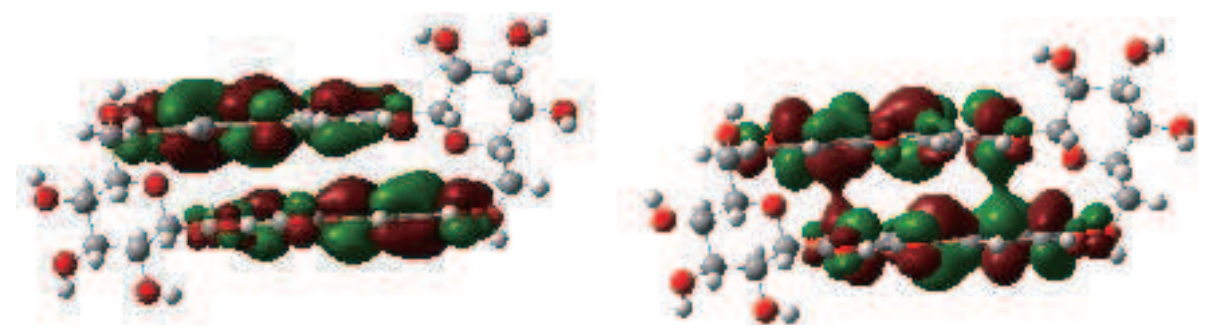

Figure 7: Highest occupied molecular orbitals and lowest unoccupied molecular orbitals of optimized mangiferin momomers.

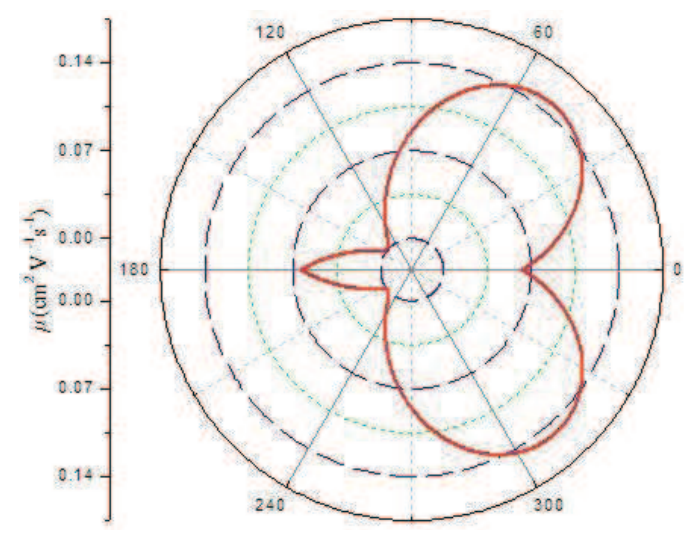

Figure 8: Angular-resolution anisotropic mobility curves of mangiferin crystal.

In order to better understand the difference of electronic couplings in mangiferin crystal, the frontier HOMOs and the LUMOs of optimized mangiferin monomers are plotted in Fig. 7, which shows strong overlaps of $\pi$ conjugating in HOMOs and LUMOs.

The diffusion coefficient and the drift mobility of holes in mangiferin crystal can be estimated from Eq. 14 using the electronic coupling and reorganization energy to calculate the mobility orientation function in the a-b plane for mangiferin. In the range of $1^{\circ} \leq \phi \leq$ $180^{\circ}, \mu_{\phi}=0.1479 \cos ^{2}\left(49.5^{\circ}-\phi\right)+0.0003 \cos ^{2}+0.0002 \cos ^{2}\left(114.75^{\circ}-\phi\right)$; and in the range of $181^{\circ} \leq \phi \leq 360^{\circ}, \mu_{\phi}=0.1479 \cos ^{2}\left(310.5^{\circ}-\phi\right)+0.0003 \cos ^{2}\left(180^{\circ}-\phi\right)+0.0002 \cos ^{2}\left(335.25^{\circ}-\phi\right)$. The anular resolution anisotropic electron-transfer mobilities in mangiferin single crystal are presented in Fig. 8. Therefore, the maximum electronic mobilities of $0.148 \mathrm{~cm}^{2} \mathrm{~V}^{-1} \mathrm{~s}^{-1}$ for mangiferin are near $49^{\circ} / 311^{\circ}$ in the angle-resolution figure. The high mobility is because of close packing of the molecules in crystal, which has the strong electronic couplings of the $\pi$-conjugated orbitals. This result confirms that mangiferin crystal is favor to function as p-type organic semiconductor, which is similar to the result of quercetin [10]. It indicates that the angle dependence of electron-transfer mobility shows remarkable anisotropic behavior. The difference can be derived from the relative magnitude of electron-transfer integrals. The distribution of angular resolution anisotropic mobility can help us to understand the charge-transport property and get high-performance organic semiconductor materials. 


\section{Conclusion}

The mechanism of action and structural requirements are studied at B3LYP / 6-311+G(d,p) level for mangiferin. The calculated BDE values for the four $\mathrm{OH}$ sites and spin density distribution results of the corresponding radicals confirm the important role of the B-ring, especially the 7-OH group in the antioxidant properties of mangiferin. It also shows that mangiferin is expected to act as hydrogen donors to reactive free radicals, convert them into more stable nonreactive species and terminate the free-radical chain reaction. The nucleophilic attacking of $\bullet \mathrm{OH}$ radical to the mangiferin leads to an intermediate that is converted directly to the stable products by releasing $39.8 \mathrm{kcal} / \mathrm{mol}$ of energy. In the addition of a $\bullet \mathrm{OOH}$ radical to the mangiferin molecule, the expected product is generated from a intermediate via a transition state. The barrier energy of this reaction is about 8.6 $\mathrm{kcal} / \mathrm{mol}$ for the $7-\mathrm{OH}$ homolytic bond cleavage. It also indicates that mangiferin has the favorable hole transport ability with the mobility as high as $0.148 \mathrm{~cm}^{2} \mathrm{~V}^{-1} \mathrm{~s}^{-1}$. On the basis of these results, we confirm that mangiferin has wide application prospects not only as antioxidant, but as promising p-type organic semiconductor material.

Acknowledgments. Much thanks to Prof. Keli Han for his help and provide the anisotropic charge transport calculation codes.

\section{References}

[1] E.C. Bate-Smith and J.B. Harborne, Mangiferin and other glycophenolics in iris species. Nature. 198 (1963), pp. 1307-1308.

[2] H.H. Liu, K. Wang, Y.H. Tang, Z.L. Sun, L.H. Jian, Z.X. Li, B. Wu and C.G. Huang, Structure elucidation of in vivo and in vitro metabolites of mangiferin. J. Pharmaceut. Biomed. 55 (2011), pp. 1075-1082.

[3] J.X. Li, M. Malakhova, M. Mottamal, K. Reddy, I. Kurinov, A. Carper, A. Langfald, N. Oi, M.O. Kim, F. Zhu, C.P. Sosa, K.Y. Zhou, A.M. Bode and Z.G. Dong, Mangiferin suppresses skin cancers induced by solar ultraviolet radiation by targeting ERK kinases. Cancer. Res. 72 (2012), pp. 260-270.

[4] F.N. Ko, C.N. Liu, S.S. Liu, T.F. Huang and C.M. Teng, Vasorelaxation of rat thoracic aorta caused by mangiferin isolated from Gentianaceae. Eur. J. Pharmacol. 192 (1991), pp. 133-139.

[5] X.Y. Duang, Q.X. Wang, D. Zhou and D.M. Huang, Mangiferin: A possible strategy for periodontal disease to therapy. medical hypotheses. 76 (2011), pp. 486-488.

[6] J.P. Wang, S.L.Raung, C.N. Lin, and C.M.Teng, Inhibitory effect of mangiferin, a xanthone from Tripterospermum lanceolatum, on cutaneous plasma extravasation. Eur. J. Pharmacol. 251 (1994), pp. 35-42.

[7] Hsu, M.F.; Raung, S.L.; Tsao, L.T.; Lin, C.N.J.P. Wang, Examination of the inhibitory effect of mangiferin in formylmethionyl-leucyl-phenylalanine-induced respiratory burst in rat neutrophils. Free. Radical. Bio. Med. 23 (1997), pp. 1035-1045.

[8] G. L. Pardo-Andreu, C. Sánchez-Baldoquín, R. Ávila-González, R. Delgado,Z. Naal and C. Curti, Fe(III) improves antioxidant and cytoprotecting activities of mangiferin. Eur. J. Pharmacol.547 (2006), pp. 31-36. 
[9] G. L. Pardo-Andreu, R. A. Cavalheiro, D. J. Dorta, Z. Naal, R. Delgado, A. E. Vercesi and C. Curti, Fe(III) shifts the mitochondria permeability transition-eliciting capacity of mangiferin to protection of organelle. The journal of pharmacology and experimental therapeutics. 320 (2007), pp. 646-653.

[10] O. Gullu and A. Turut, Photovoltaic and electronic properties of quercetin/p-InP solar cells. Sol. Energ. Mater. Sol. C. 92 (2008), pp. 1205-1210.

[11] Y.M. Sung, F.C. Hsu, C.T. Chen, W.F. Su and Y.F. Chen, Enhanced photocurrent and stability of inverted polymer/ZnO-nanorod solar cells by 3-hydroxyflavone additive. Sol. Energ. Mater. Sol. C. 98 (2012), pp. 103-109.

[12] B. Halliwell, Dietary polyphenols: good, bad, or indifferent for your health. Cardiovasc. Res. 73 (2007), pp. 341-347.

[13] P.G. Pietta, Flavonoids as antioxidants. J. Nat. Prod. 63 (2000), pp. 1035-1042.

[14] C. Manach, A. Mazur and A. Scalbert, Polyphenols and prevention of cardiovascular diseases. Curr. Opin. Lipidol. 16 (2005), pp. 77-84.

[15] P.M. Aron and J.A. Kennedy, Flavan-3-ols: Nature, occurrence and biological activity. Mol. Nutr. Food. Res. 52 (2008), pp. 79-104.

[16] W.I. Rosiane, Z.D. Oliveira, S.C. Fernandes and I.C. Vieira, Development of a biosensor based on gilo peroxidase immobilized on chitosan chemically crosslinked with epichlorohydrin for determination of rutin. J. Pharm. Biomed. Anal.41 (2006), pp. 366-372.

[17] J. Lengyel, J. Rimarčík, A. Vagánek and E. Klein, On the radical scavenging activity of isoflavones: thermodynamics of O-H bond cleavage. Phys. Chem. Chem. Phys. 15 (2013), pp. 10895-10903.

[18] A. Vagánek, J. Rimarčík, V. Lukeš and E. Klein, On the energetics of homolytic and heterolytic O-H bond cleavage in flavonoids. Comput. Theor. Chem. 99 (2012), pp. 1192-200.

[19] G. Mina and M.H. Majid, Quantum mechanical study of antioxidative ability and antioxidative mechanism of rutin (vitamin P) in solution. Carbohydr. Res. 346 (2011), pp. 739-744.

[20] M. Leopoldini, I.P. Pitarch, N.Russo and M.J. Toscano, Structure, conformation, and electronic properties of apigenin, luteolin, and taxifolin antioxidants. A first principle theoretical study. J. Phys. Chem. A. 108 2004, pp. 92-96.

[21] M. Watanabe, Y. Ohshita and T. Tsuchida, Antioxidant compounds from buckwheat (Fagopyrum esculentum Möench) hulls. J. Agric. Food. Chem. 45 (1997), pp. 1039-1044.

[22] H.J. Xie, Q.F. Lei, and W.J. Fang, Density functional theory study on the antioxidation activity of quercetin. Acta. Chim. Sinica. 68 (2010), pp. 1467-1472.

[23] M. Rossi, L.F. Rickles and W.A. Halpin, The crystal and molecular structure of quercetin: A biologically active and naturally occurring flavonoid. Biol. Chem. 14 (1986), pp. 55-69.

[24] M.R. Narayan, Review: Dye sensitized solar cells based on natural photosensitizers. Renew. Sust. Energ. Rev. 16 (2012), pp. 208-215.

[25] R.L. Headrick, S. Wo, F. Sansoz and J.E. Anthony, Anisotropic mobility in large grain size solution processed organic semiconductor thin films. Appl. Phys. Lett. 92 (2008), pp. 063302063303.

[26] C. Reese and Z.N. Bao, High-resolution measurement of the anisotropy of charge transport in single crystals. Adv. Mater. 19 (2007), pp. 4535-4538.

[27] S.C.B. Mannsfeld, J. Locklin, C. Reese, M.E. Roberts, A.J .Lovinger and Z.N. Bao, Probing the anisotropic field-effect mobility of solution-deposited dicyclohexyl- $\alpha$-quaterthiophene single crystals. Adv. Funct. Mater. 17 (2007), pp. 1617-1622.

[28] V.C. Sundar, J. Zaumseil, V. Podzorov, E. Menard, R.L. Willett, T. Someya, M. E. Gershenson and J.A. Rogers, Elastomeric transistor stamps: Reversible probing of charge transport in 
organic crystals. Science. 303 (2004), pp. 1644-1646.

[29] J.Y. Lee, S. Roth and Y.W. Park, Anisotropic field effect mobility in single crystal pentacene. Appl. Phys. Lett. 88 (2006), pp. 252106.

[30] M.M. Ling, C. Reese, A.L. Briseno and Z.N. Bao, Non-destructive probing of the anisotropy of field-effect mobility in the rubrene single crystal. Synth. Met. 157 (2007), pp. 257-260.

[31] N.E. Gruhn, D.A. da Silva Filho, T.G. Bill, M. Malagoli, V. Coropceanu, A. Kahn, J.L. Brédas, The vibrational reorganization energy in pentacene: Molecular influences on charge transport. J. Am. Chem. Soc. 124 (2002), pp. 7918-7919.

[32] F. Amy, C. Chan and A. Kahn, Polarization at the gold/pentacene interface. Org. Electron. 6 (2005), pp. 85-91.

[33] R. Ruiz, B. Nickel, N. Koch, L.C. Feldman, R.F. Haglund, A. Kahn and G. Scoles, Pentacene ultrathin film formation on reduced and oxidized Si surfaces. Phys. Rev. B. 67 (2003), pp. 125406.

[34] C.K. Chan, E.G. Kim, J.L. Brédas and A. Kahn, Molecular n-type doping of 1,4,5,8naphthalene tetracarboxylic dianhydride by pyronin B studied using direct and inverse photoelectron spectroscopies. Adv. Funct. Mater. 16 (2006), pp. 831-837.

[35] C.K. Chan and A. Kahn, N-doping of pentacene by decamethylcobaltocene. Appl. Phys. A: Mater. Sci. Process. 95 (2009), pp. 7-13.

[36] I.G. Hill, J. Hwang, A. Kahn, C. Huang, J.E. McDermott and J. Schwartz, Energy level alignment between 9-phosphonoanthracene self-assembled monolayers and pentacene. Appl. Phys. Lett. 90 (2007), pp. 012109.

[37] S.W. Yin and Y.F. Lv, Modeling hole and electron mobilities in pentacene ab-plane. Org. Electron. 9 (2008), pp. 852-858.

[38] K. Hannewald and P.A. Bobbert, Anisotropy effects in phonon-assisted charge-carrier transport in organic molecular crystals. Phys. Rev. B. 69 (2004), pp. 075212.

[39] Y.K. Lan and C.I. Huang, A theoretical study of the charge transfer behavior of the highly regioregular poly-3-hexylthiophene in the ordered state. J. Phys. Chem. B. 112 (2008), pp. 14857-14862.

[40] G.A. de Wijs, C.C. Mattheus, R.A. de Groot and T.T.M. Palstra, Anisotropy of the mobility of pentacene from frustration. Synth. Met. 139 (2003), PP. 109-114.

[41] N.S. Hush, Chem. J. Phys. 28 (1958), pp. 962-972.

[42] R.A. Marcus, On the theory of oxidation-Reduction Reactions Involving Electron Transfer. I. J. Chem. Phys. 24 (1956), pp. 966-978.

[43] S.H. Wen, A. Li, J.L. Song, W.Q. Deng, K.L. Han and W.A. Goddard, First-principles investigation of anistropic hole mobilities in organic semiconductors. J. Phys. Chem. B. 113 (2009), pp. 8813-8819.

[44] S.H. Wen, W.Q. Deng and K.L. Han, Revealing quantitative structure-activity relationships of transport properties in acene and acene derivative organic materials. Phys. Chem. Chem. Phys. 12 (2010), pp. 9267-9275.

[45] J.D. Huang, S.H. Wen, W.Q. Deng and K.L. Han, Simulation of Hole Mobility in $\alpha$-Oligofuran Crystals. J. Phys. Chem. B. 115 (2011), pp. 2140-2147.

[46] S.H. Wen, W.Q. Deng and K.L. Han, Ultra-low resistance at TTF-TCNQ organic interfaces. Chem. Commun. 46 (2010), pp. 5133-5135.

[47] S. Chai, S.H. Wen and K.L. Han, Understanding electron-withdrawing substituent effect on structural, electronic and charge transport properties of perylene bisimide derivatives. Org. Electron. 12 (2011), pp. 1806-1814.

[48] J. Huang, S. Chai, S. Wen, W. Deng and K. Han, Anisotropic Mobilities in Organic Semicon- 
ductors. Nature Protocol Exchange (2013) doi:10.1038/protex. 070 (2013).

[49] X.Y. Zhang and G.J. Zhao, Anisotropic Charge Transport in Bisindenoanthrazoline-Based n-Type Organic Semiconductors. J. Phys. Chem. C. 116 (2012), pp. 13858-13864.

[50] J.S. Binkley, J.A. Pople and W.J. Hehre, Self-consistent molecular orbital methods. 21. Small split-valence basis sets for first-row elements. J. Am. Chem. Soc. 102 (1980), pp. 939-947.

[51] M. Namazian, H.R. Zare and M.L. Coote, Determination of the absolute redox potential of Rutin: Experimental and theoretical studies. Biophys. Chem. 132 (2008), pp.64-68.

[52] P. Trouillas, D. Marsal, R. Siri, J. Lazzaroni and L. Duroux, A DFT study of the reactivity of $\mathrm{OH}$ groups in quercetin and taxifolin antioxidants: The specificity of the 3-OH site. Food. Chem. 97 (2006), pp. 679-688.

[53] H.F. Ji, G.Y. Tang and H.Y. Zhang, Theoretical elucidation of DPPH radical-scavenging activity difference of antioxidant xanthones. QSAR Comb. Sci. 24 (2005), pp. 826-830.

[54] S. Erkoc, F. Erkoc and N.J. Keskin, Theoretical investigation of quercetin and its radical isomers. J. Mol. Struct. Theochem. 631 (2003), pp. 141-146.

[55] H.F. Ji and L. Shen, A DFT study on deactivation of triplet excited state riboflavin by polyphenols. Int. J. Mol. Sci. 9 (2008), pp. 1908-1914.

[56] A.M. Mendoza-Wilson and D. Glossman-Mitnik, Theoretical study of the molecular properties and chemical reactivity of (+)-catechin and (-)-epicatechin related to their antioxidant ability. Mol. Struct. Theochem. 761 (2006), pp. 97-106.

[57] M.J. Frisch, G.W. Trucks and H.B. Schlegel, Gaussian 03, revision C. 02; Gaussian, Inc. Wallingford, CT. 26 (2004).

[58] C.Y. Hou, X.F. Chen, J.Y. Liu and B.Z. Wang, A density functional theory study of the synthesis of 5-chloro-benzofuroxan from 5-chloro-2-nitroaniline. Acta. Chim. Sin. 9 (2011), pp. 1065-1069.

[59] C.Y. Hou, H.X. Zhang and C.C. Sun, $A b$ initio study of the spectroscopy of $\mathrm{CH}_{3} \mathrm{~N}$ and $\mathrm{CH}_{3} \mathrm{CH}_{2}$ N. J. Phys. Chem. A. 110 (2006), pp. 10260-10266.

[60] C.Y. Hou, X.F. Chen, J.Y. Liu, W.P. Lai and B.Z. Wang, DFT study of benzofuroxan synthesis mechanism from 2-Nitroaniline via sodium hypochlorite. Chin. J. Chem. Phys. 23 (2010), pp. 387-392.

[61] J.J. Kwiatkowski, J. Nelson, H. Li, J.L. Brédas, W. Wenzel and C. Lennartz, Simulating charge transport in tris (8-hydroxyquinoline) aluminium (Alq3). Phys. Chem. Chem. Phys. 10 (2008), pp. 1852-1858.

[62] T. Yamada, T. Sato, K. Tanaka and H. Kaji, Percolation paths for charge transports in $\mathrm{N}, \mathrm{N}^{\prime}-$ diphenyl-N,N'-di(m-tolyl)benzidine (TPD). Org. Electron. 11 (2010), pp. 255-265.

[63] J.L. Brédas, D. Beljonne, V. Coropceanu and J. Cornil, Charge-transfer and energy-transfer processes in $\pi$-conjugated oligomers and polymers: A molecular picture. Chem. Rev. 104 (2004), pp. 4971-5004.

[64] G.R. Hutchison, M.A. Ratner and T.J. Marks, Hopping transport in conductive heterocyclic oligomers: reorganization energies and substituent effects. J. Am. Chem. Soc. 127 (2005), pp. 2339-2350.

[65] V. Coropceanu, T. Nakano, N.E.Gruhn, O. Kwon, T. Yade, Ki. Katsukawa and J.L. Brédas, Probing charge transport in $\pi$-stacked fluorene-based systems. J. Phys. Chem. B. 110 (2006), pp. 9482-9487.

[66] K. Senthilkumar, F.C. Grozema, F.M. Bickelhaupt and L.D.A.Siebbeles, Charge transport in columnar stacked triphenylenes: Effects of conformational fluctuations on charge transfer integrals and site energies. J. Chem. Phys. 119 (2003), pp. 9809-9817.

[67] E.F. Valeev, V. Coropceanu, D.A. da Silva Fihlo, S. Salman and J.L. Brédas, Effect of electronic 
polarization on charge-transport parameters in molecular organic semiconductors. J. Am. Chem. Soc. 128 (2006), pp. 9882-9886.

[68] T. Lu and F.W. Chen, Multiwfn: A multifunctional wavefunction analyzer. J. Comput. Chem. 33 (2012), pp. 580-592.

[69] R.A. Marcus, Electron transfer reactions in chemistry. Theory and experiment. Rev. Mod. Phys. 65 (1993), pp. 599-610.

[70] R.A. Marcus, On the theory of oxidation-reduction reactions involving electron transfer. v. comparison and properties of electrochemical and chemical rate constants. J. Phys. Chem. 67 (1963), pp. 853-857.

[71] W.Q. Deng and W.A.III. Goddard, Predictions of hole mobilities in oligoacene organic semiconductors from quantum mechanical calculations. J. Phys. Chem. B. 108 (2004), pp. 86148621.

[72] V. Coropceanu, J. Cornil, D.A. da Silva Filho, Y. Olivier, R. Silbey and J.L. Brédas, Charge transport in organic semiconductors. Chem. Rev. 107 (2007), pp. 926-952.

[73] G.R. Hutchison, M.A. Ratner and T.J. Marks, Intermolecular charge transfer between heterocyclic oligomers. effects of heteroatom and molecular packing on hopping transport in organic semiconductors. J. Am. Chem. Soc. 127 (2005), pp. 16866-16881.

[74] C.L. Wang, F.H.Wang, X.D. Yang, Q.K. Li and Z.G. Shuai, Theoretical comparative studies of charge mobilities for molecular materials: Pet versus bnpery. Org. Electron. 9 (2008), pp. 635-640.

[75] H. Moon, R. Zeis, E.J. Borkent, C. Besnard, A.J. Lovinger, T. Siegrist, C. Kloc and Z.N. Bao, Synthesis, Crystal Structure, and Transistor Performance of Tetracene Derivatives. J. Am. Chem. Soc. 126 (2004), pp. 15322-15323.

[76] M.Y. Kuo, H.Y. Chen and I. Chao, Cyanation: Providing a Three-in-One Advantage for the Design of n-Type Organic Field-Effect Transistors. Chem. Eur. J. 13 (2007), pp. 4750-4758.

[77] J.B. Foresman and E.Frisch, Exploring chemistry with electronic structure methods, 2nd ed., Gaussian Inc, Pittsburgh, USA, (1996).

[78] J. Andres and J. Bertran, Química teórica computacional, Primera edición universitat Jaume I, Spain, (2000).

[79] C.Y. Hou, Theoretical study of antioxidative ability and antioxidative mechanism of norathyriol in solution. Comp. Theor. Chem. 1028 (2014), pp. 87-91.

[80] A. Szabo and N.S. Ostlund, Modern quantum chemistry: Introduction to advanced electronic structure theory,Dover Publications, New York, (1982).

[81] J.P. Christopher, M.M. Paul and R. Leo, An assessment of theoretical procedures for the calculation of reliable radical stabilization energies. J. Chem. Soc. Perkin Trans. 2 (1999), pp. 2305-2313.

[82] G.J. Zhao, J.Y. Liu, L.C. Zhou and K.L. Han, Site-selective photoinduced electron transfer from alcoholic solvents to the chromophore facilitated by hydrogen bonding: A new fluorescence quenching mechanism. J. Phys. Chem. B. 111(30) (2007), pp. 8940-8945.

[83] G. J. Zhao and K. L.Han, Site-specific solvation of the photoexcited protochlorophyllide a in methanol: Formation of the hydrogen-bonded intermediate state induced by hydrogenbond strengthening. Biophys. J. 94(1) (2008), pp. 38-46. 